\title{
Enhanced dispersion mapping for OPC-aided transmission systems
}

Kaminski, Pawel Marcin; Da Ros, Francesco; Clausen, Anders; Forchhammer, Søren; Oxenløwe, Leif Katsuo; Galili, Michael

\section{Published in:}

Proceedings of the 45th European Conference on Optical Communication

Link to article, DOI:

10.1049/cp.2019.1135

Publication date:

2020

Document Version

Peer reviewed version

Link back to DTU Orbit

\section{Citation (APA):}

Kaminski, P. M., Da Ros, F., Clausen, A., Forchhammer, S., Oxenløwe, L. K., \& Galili, M. (2020). Enhanced dispersion mapping for OPC-aided transmission systems. In Proceedings of the 45th European Conference on Optical Communication (pp. 1498-1502). Institution of Engineering and Technology.

https://doi.org/10.1049/cp.2019.1135

\section{General rights}

Copyright and moral rights for the publications made accessible in the public portal are retained by the authors and/or other copyright owners and it is a condition of accessing publications that users recognise and abide by the legal requirements associated with these rights.

- Users may download and print one copy of any publication from the public portal for the purpose of private study or research.

- You may not further distribute the material or use it for any profit-making activity or commercial gain

- You may freely distribute the URL identifying the publication in the public portal 


\title{
ENHANCED DISPERSION MAPPING FOR OPC-AIDED TRANSMISSION SYSTEMS \\ Pawel M Kaminski*, Francesco Da Ros, Anders T Clausen, Soren Forchhammer, Leif K Oxenlowe, and Michael Galili
}

\author{
DTU Fotonik, Technical University of Denmark, DK-2800 Kgs. Lyngby, Denmark \\ *pkam@fotonik.dtu.dk
}

Keywords: LINK DESIGN, OPTICAL SIGNAL PROCESSING, NONLINEARITY COMPENSATION

\begin{abstract}
We present a novel approach to designing transmission links with lumped amplification achieving near optimal nonlinearity compensation by inclusion of an inline OPC. SNR gains of $6.0 \mathrm{~dB}$ to $7.5 \mathrm{~dB}$ are found numerically in WDM multi-span transmission when comparing to standard EDFA-based systems.
\end{abstract}

\section{Introduction}

With the ever increasing traffic demands, present optical communication systems are now struggling to meet the future requirements in terms of throughput, cost-efficiency and reliability. The inherent fiber nonlinearity has imposed limits on the achievable information rates and transmission distances because of the additional signal degradations, which present-day systems fail to address [1]. Nonlinearity suppression and compensation techniques have therefore been attracting a lot of attention, focusing on both digital and optical approaches. Digital methods typically require significant processing times, but have been shown to provide efficient compensation within the analog bandwidth of the receiver [2], which however limits its practical effectiveness to a single channel. On the other hand, all-optical techniques are nearly instantaneous and can address the nonlinear distortions across all frequency channels in wavelengthdivision multiplexed (WDM) systems. Among the optical approaches, optical phase-conjugation (OPC) has been demonstrated to limit the distortion [3-6], but its performance gain relies heavily on specific link properties to achieve efficient nonlinearity compensation. In particular, identical nonlinear phase shifts $\Phi_{N L}=\gamma P(z, t) L$ must occur on both sides of the OPC for the same pulse shapes of the signal and the conjugated copy (idler), respectively. This requirement approximately translates into power and dispersion symmetry with respect to the point of conjugation, with the perfect compensation achieved when the power is identical at the opposite values of the accumulated dispersion. The degree of symmetry, and thus OPC effectiveness, can then be evaluated by means of power vs. accumulated dispersion diagrams (PADD) $[7,8]$. The typical approach to satisfy the symmetry conditions relies on tailoring power profiles to match the profile of accumulated dispersion. Despite impressive demonstrations of OPC gains for short Raman-amplified links [6], most practical systems fail to satisfy the strict compensation criteria.

Instead of manipulating the power profiles along the link, in this work, we fully rely on dispersion engineering to effectively maximize the symmetry for standard EDFA- amplified systems with exponential power decay. We show that perfectly symmetric PADD can be achieved with a careful, yet simple dispersion map, greatly enhancing OPC compensation efficiency and overall system performance. The results for the optimum mapping are compared against other systems with different dispersion profiles for reference. The simulated links ultimately fall into three distinct categories: dispersion uncompensated, dispersion compensated, and dispersion managed. We evaluate the performance of each scheme, demonstrate possible improvements from OPC, and justify its origin in terms of the symmetry in power and temporal shape. This systematic analysis has been conducted for two-span links, but the same reasoning is also extended to multi-span systems and the multi-span results show similar trends. 16-quadrature amplitude modulation (QAM) signals at $16-\mathrm{GBd}$ are employed for the study, and the standard split-step Fourier method (SSFM) is used for the numerical simulations.

\section{Simulation Setup}

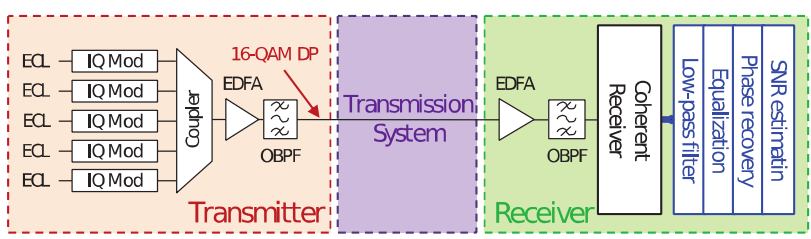

Fig. 1 Numerical setup of the transmitter and the receiver employed for the performance evaluation of the link designs.

The numerical setup employed for the analysis is sketched in Fig. 1. At the transmitter site, up to five WDM channels on a $20 \mathrm{GHz}$ grid are generated around $1550 \mathrm{~nm}$ (carrier linewidth $v=10 \mathrm{kHz}$ ). Each channel is modulated with distinct 16-QAM data at $16 \mathrm{GBd}$ per polarization. The channels are combined, amplified and filtered before entering the transmission systems under test. At the end of the transmission, the signals are amplified to a constant output power, and received with a coherent receiver followed by standard digital signal processing (DSP), including equalization, phase recovery and effective signal-to-noise 

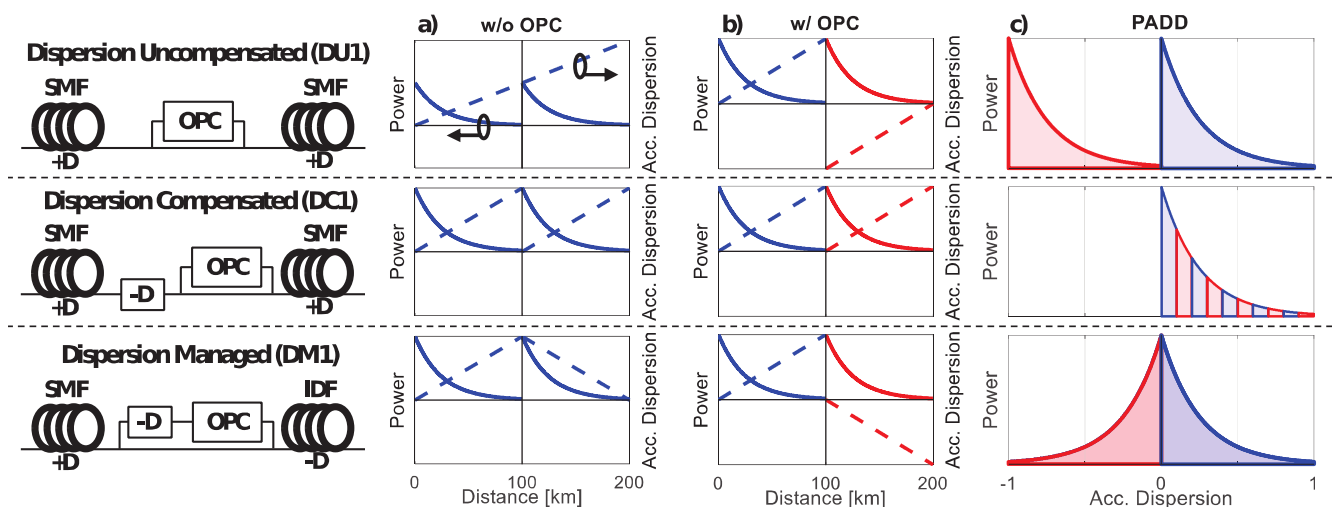

Fig. 2 Transmission systems under test with the corresponding: power (solid) and dispersion (dashed) evolution in distance without (a) and with (b) mid-span OPC. (c) Power vs. accumulated dispersion diagrams attached alongside. Signal and idler properties marked in blue and red, respectively.

ratio (SNR) estimation. As in [9], the effective SNR is estimated from the transmitted and received QAM symbols, $x_{k}$ and $y_{k}$, after the full DSP chain according to: $S N R_{\text {eff }}=$ $\mathbb{E}_{k}\left[\left|x_{k}\right|^{2}\right] / \mathbb{E}_{k}\left[\left|y_{k}-x_{k}\right|^{2}\right]$, where $\mathbb{E}[\cdot]$ denotes the expectation operation.

\section{Link Designs}

Three main types of transmission link designs are considered, as indicated in Fig. 2. Each system consists of two 100-km spans, with the standard single mode fiber (SSMF) parameters employed for the simulations: fiber loss $\alpha=$ $0.2 \mathrm{~dB} / \mathrm{km}$, group velocity dispersion $D=17 \mathrm{ps} / \mathrm{nm} / \mathrm{km}$, nonlinear coefficient $\gamma=1.3 \mathrm{~W} / \mathrm{km}$. Amplifier noise figures are set to $N F=5 \mathrm{~dB}$. In order to focus on the link designs, OPC is emulated with an ideal conjugation of the complex field amplitude with no penalties associated with the process: $A(z, t) \rightarrow A^{*}(z, t)$. Higher order dispersion and polarization mode dispersion (PMD) have been neglected in this analysis to allow focusing on the PADD.

The first scenario is the most standard EDFA-amplified dispersion uncompensated (DU1) system. Including OPC in the middle reverses the sign of nonlinearity and accumulated dispersion, leading to asymmetric PADD (Fig 2, DU1, c) with high power at low positive dispersion for signal and high power at high negative dispersion for idler, therefore it is known to lead to very limited OPC gains [3]. The second scenario also transmits over SSMF, but it includes dispersion compensating modules after each span (DC1). This resets the accumulated dispersion to zero, and thus it remains at zero upon conjugation. The corresponding PADD (Fig 2, DC1, c) is still very asymmetric, although some power-dispersion matching exists close to zero accumulated dispersion (high power at zero dispersion for both signal and idler), potentially improving the gains from OPC [4]. Finally, the third scenario is a dispersion managed (DM1) system, which is built from SSMF in the first span and inverse dispersion fiber (IDF: parameters as SSMF except for $D=-17 \mathrm{ps} / \mathrm{nm} / \mathrm{km}$ ) in the second span. For the OPC case, a supplementary dispersion compensating module is placed after the first span, such that the accumulated dispersion remains zero upon conjugation. Although the resultant power/dispersion versus distance graph does not appear symmetric, the corresponding PADD
(Fig 2, DM1, c) matches the ideal profiles for the maximum OPC gains, with identical powers for positive (signal) and negative (idler) values of accumulated dispersion. For complete nonlinearity cancellation, the idler must undergo the dispersive and nonlinear evolution in the reverse order of how the signal has accumulated the distortions (i.e. pulse propagation must be symmetric). Despite ideal PADD, the propagation is not symmetric in DM1, as the impairments at the beginning of the first span are compensated at the beginning of the second span. Although the nonlinearity is effectively cancelled in the wrong order, major improvement of performance is still expected as shown below.

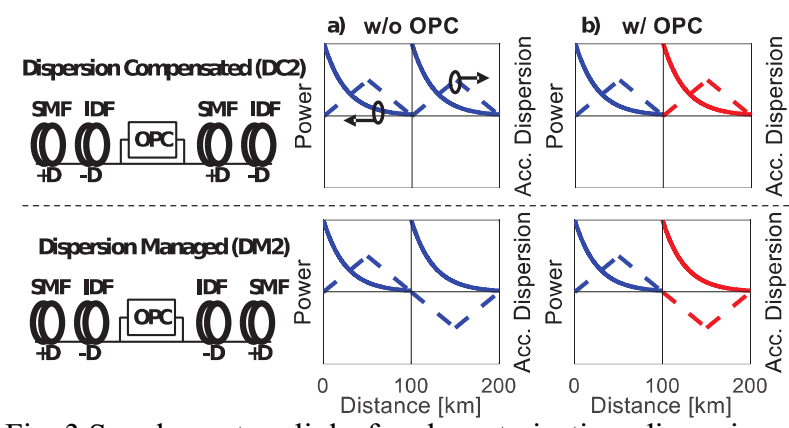

Fig. 3 Supplementary links for characterization: dispersion compensated (DC2) and dispersion managed (DM2) systems.

It is noted that there exists a number of variations of dispersion compensated and dispersion managed links. Examples of such are illustrated in Fig. 3. DC2 and DM2 are both made of composite SSMF+IDF spans for complete dispersion compensation of each transmission span. The accumulated dispersion map in DC2 is positive for all spans, leading to PADD resembling DC1 with limited symmetry properties. On the other hand, in DM2 the order of SSMF and IDF is inverted after the OPC yielding identical signal and idler powers occurring at the opposite values of accumulated dispersion, thus leading to a perfectly symmetric PADD like in DM1, and therefore high OPC gains. Moreover, the two links require no extra dispersion compensating modules, are identical with regard to the amount and type of the fiber used, and can be interchanged simply by reversing the fiber of the second span. Despite the similarities, the performance with OPC included is expected to vary greatly. 


\section{Simulation Results}

The system performance is evaluated in terms of effective receiver SNR as a function of total launched signal power into each of the two transmission spans. All five scenarios have been simulated with and without mid-link OPC, and Fig. 4 compares the results for single- and five-channel transmission.

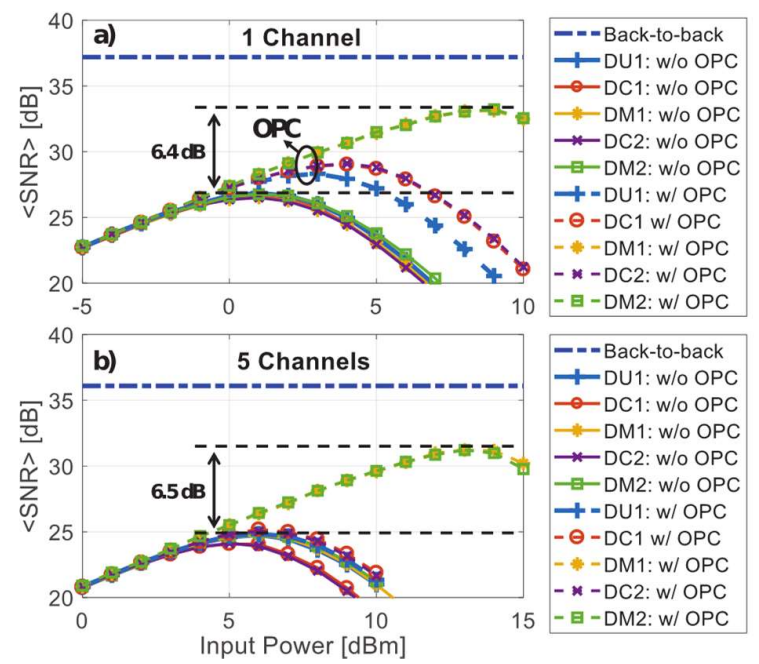

Fig. 4 Effective receiver SNR versus total launched power for 2-span systems without (solid) and with (dashed) OPC for a single channel (a) and five WDM channels (b).

In a single channel scenario (Fig 4a), all the systems without OPC are shown to have a comparable performance, with the dispersion uncompensated transmission (DU1) slightly outperforming the others. The systems with OPC vary greatly due to different symmetry properties of the spans. They all, however, achieve SNR improvements due to the addition of OPC. The dispersion uncompensated system (DU1) offers the least improvement $(1.5 \mathrm{~dB})$ due to poor power-dispersion symmetry as indicated in the corresponding PADD (Fig. 2). The dispersion compensated systems (DC1 and DC2) result in higher gains $(2.5 \mathrm{~dB})$ due to power-dispersion matching at the beginning of the spans where the nonlinearity is the strongest. Nonetheless, the dispersion managed systems (DM1 and DM2) clearly outperform the rest for the case with OPC. Because of symmetry in PADD, the nonlinear phaseshifts in the two spans are matched for signal and idler, respectively, and high gains $(6.4 \mathrm{~dB})$ are achieved. Similar trends are observed for the WDM case (Fig. 4b), but the difference between the OPC systems under test is even more pronounced. The dispersion uncompensated transmission (DU1) is now superior to other setups without OPC. However, for the OPC scenarios, very little gain is observed for dispersion uncompensated (DU1) and dispersion compensated (DC1 and DC2) transmission due to poor PADD symmetry. The broader bandwidth of WDM signals compared to a single channel leads to larger changes in temporal waveform with accumulated dispersion, increasing the importance of PADD for OPC compensation. As a result, only the dispersion managed systems (DM1 and DM2) with a high degree of symmetry, will offer significant gains and superior performance (6.5 dB increase over DU1).

\section{$5 \quad$ Multi-Span Systems}

The analysis is extended to multiple spans for the three general scenarios of dispersion uncompensated, dispersion compensated, and dispersion managed transmission. The scenarios under analysis are depicted in Fig. 5. It is noted that many variations of each type of system exist, but they exhibit comparable performance due to similarities in PADD.

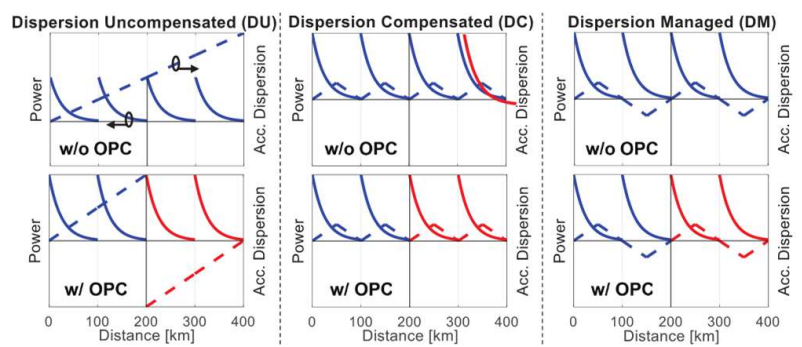

Fig. 5 Transmission systems for multi-span analysis: dispersion uncompensated (DU), dispersion compensated (DC), and dispersion managed (DM). Four spans shown only.

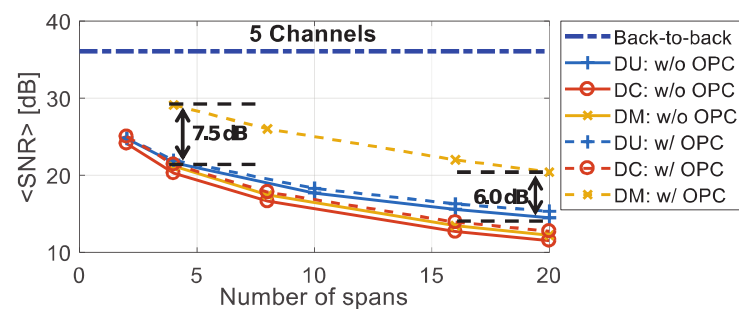

Fig. 6 Effective SNR at optimum launched power without (solid) and with (dashed) OPC for a varying number of spans.

Fig. 6 shows the maximum achievable SNR at optimum launched power. The dispersion uncompensated setup (DU) outperforms the other scenarios for the case without OPC. Regardless of the gain that mid-link OPC provides, the dispersion compensated setup (DC) with OPC is still worse than dispersion uncompensated scheme (DU) without OPC. However, by tailoring the dispersion (DM), significant gains can be achieved with OPC, clearly outperforming all the other scenarios with SNR gains of up to $6.0 \mathrm{~dB}$ and $7.5 \mathrm{~dB}$.

\section{Conclusion}

Here, we numerically evaluated the link design for EDFAamplified systems with regard to different dispersion profiles, and discussed its impact on the gains provided by OPC through PADD. We tailored the dispersion profiles to the exponential power decay of typical transmission spans, and proposed a simple dispersion managed system for improved OPC-based nonlinearity compensation. This system is shown to be superior by $6.0 \mathrm{~dB}$ to $7.5 \mathrm{~dB}$ in $\mathrm{SNR}$ with respect to other EDFA-based link configurations. We believe this is an important novel approach to designing systems achieving close to ideal nonlinearity compensation without the need for complex distributed amplification schemes.

\section{Acknowledgements}

The work was supported by the DNRF Research CoE, SPOC (ref. DNRF123), and the ERC CoG FRECOM (771878). 


\section{References}

[1] Essiambre, S., Tkach, R.W.: 'Capacity Trends and Limits of Optical Communication Networks', Proc. IEEE., 2012, 100, (5), pp. 1035-1055

[2] Ip, E., Kahn, J.M.: 'Compensation of dispersion and nonlinear impairments using digital backpropagation', Journal of Lightwave Technology, 2008, 26, (20), pp. 3416-3425

[3] Da Ros, F., Yankov, M.P., da Silva, E.P. et al., 'LinkPlacement Characterization of Optical Phase Conjugation for Nonlinearity Compensation', Proc. OFC, 2018, paper W3E.3 [4] Da Ros, F., Sackey, I., Elschner, R. et al.: 'Kerr nonlinearity compensation in a $5 \times 28-G B d$ PDM $16-\mathrm{QAM}$ WDM system using fiber-based optical phase conjugation'. Proc. ECOC, 2014, paper P.5.3

[5] Ellis, A.D., Tan, M., Iqbal, M.A. et al.: '4 Tb/s Transmission Reach Enhancement Using 10400 Gb/s SuperChannels and Polarization Insensitive Dual Band Optical Phase Conjugation', Journal of Lightwave Technology, 2016, 34, (8), pp. 1717-1723

[6] Ellis, A.D., Zhao, J., Cotter, D.: 'Approaching the nonlinear Shannon limit ', Journal of Lightwave Technology, 2016, 28, (4), pp. 423-433

[7] Minzioni, P.: 'Nonlinearity Compensation in a FiberOptic Link by Optical Phase Conjugation', Fiber and Integrated Optics, 2009, 28, (3), pp. 179-209

[8] Minzioni, P., Schiffini, A.: 'Unifying theory of compensation techniques for inrachannel nonlinear effects ', Optics Express, 2005, 13, (21), pp. 8460-8468

[9] Yankov, M.P, Da Ros, F., da Silva, E.P. et al.: 'Constellation shaping for WDM systems using 256QAM / 1024QAM with probabilistic optimization limit ', Journal of Lightwave Technology, 2016, 34, (22), pp. 5146-5156 